\title{
VARIANTS OF ANDO-HIAI TYPE INEQUALITIES FOR DEFORMED MEANS AND APPLICATIONS
}

\author{
MOHSEN KIAN $^{1}$, M. S. MOSLEHIAN ${ }^{2}$ and YUKI SEO ${ }^{3}$
}

\begin{abstract}
For an $n$-tuple of positive invertible operators on a Hilbert space, we present some variants of Ando-Hiai type inequalities for deformed means from an $n$-variable operator mean by an operator mean, which is related to the information monotonicity of a certain unital positive linear map. As an application, we investigate the monotonicity of the power mean from the deformed mean in terms of the generalized Kantorovich constants under the operator order. Moreover, we improve the norm inequality for the operator power means related to the Log-Euclidean mean in terms of the Specht ratio.
\end{abstract}

\section{INTRODUCTION}

In 2004, Ando et al. [2] succeeded in the formulation of the geometric mean for $n(\geq 3)$ positive definite matrices, and they showed that it has many required properties as the geometric mean. Yamazaki [29] pointed out that it can be extended to the positive invertible operators on a Hilbert space. Since then, many researchers have studied operator geometric means of $n$ positive invertible operators on a Hilbert space. On the other hand, Moakher [21] and then Bhatia and Holbrook [4] suggested a new definition of the geometric mean for $n$ positive definite matrices by taking the mean to be the unique minimizer of the sum of squares of distances. Computing appropriate derivatives as in [21,3] yields that it coincides with the unique positive definite solution of the Karcher equation. The unique solution of the Karcher equation is called the Karcher mean of $n$ positive definite matrices. In 2012, Lim and Pálfia [20] constructed a family of matrix power means, each with numerous desirable properties such as monotonicity, that converges to the Karcher mean and showed that these properties are preserved in the limit. Moreover, in 2014, Lawson and Lim [19] showed that the Karcher equation has a unique positive invertible solution in the infinite-dimensional setting.

2010 Mathematics Subject Classification. Primary 47A64; Secondary 47A63, 47B65.

Key words and phrases. Ando-Hiai inequality; operator mean; deformed mean; operator power mean; Karcher mean; generalized Kantorovich constant; Specht ratio. 
The so-called Golden-Thompson inequality provides a relation between matrix exponential functions. In separate works, Golden [12] and Thompson [25] proved that $\operatorname{Tr} e^{H+K} \leq \operatorname{Tr} e^{H} e^{K}$ for all Hermitian matrices $H, K$. An extension of this inequality asserts that $\left\|e^{H+K}\right\| \leq\left\|e^{H / 2} e^{K} e^{H / 2}\right\|$ holds for every unitarily invariant norm. As a complementary to the Golden-Thompson inequality, Ando and Hiai [1] presented an inequality for operator means of positive definite matrices. It is called the Ando-Hiai inequality (see also [5, 6, 28]): For each $\alpha \in(0,1]$

$$
A \sharp_{\alpha} B \leq I \quad \Longrightarrow \quad A^{r} \sharp_{\alpha} B^{r} \leq I \quad \text { for all } r \geq 1 \text {, }
$$

where the weighted geometric mean is defined by

$$
A \sharp_{\alpha} B=A^{1 / 2}\left(A^{-1 / 2} B A^{-1 / 2}\right)^{\alpha} A^{1 / 2}
$$

for positive invertible operators $A$ and $B$. The Ando-Hiai inequality is not only a significant inequality in the operator theory [7], but also plays an essential role in the quantum information theory, quantum statistics, and so on; see [9, 23]. The extension of this inequality to the Karcher mean was established by Yamazaki [30]: For each probability vector $\omega=\left(\omega_{1}, \ldots, \omega_{n}\right)$

$$
G_{\omega}\left(A_{1}, \ldots, A_{n}\right) \leq I \quad \Longrightarrow \quad G_{\omega}\left(A_{1}^{r}, \ldots, A_{n}^{r}\right) \leq I \quad \text { for all } r \geq 1,
$$

where the Karcher mean $G_{\omega}\left(A_{1}, \ldots, A_{n}\right)$ for positive invertible operators $A_{1}, \ldots, A_{n}$ is defined to be the unique solution of the Karcher equation

$$
\sum_{j=1}^{n} \omega_{j} \log \left(X^{-1 / 2} A_{j} X^{-1 / 2}\right)=0
$$

and its modification for the operator power means was also shown by Wada [27].

Recently, Hiai et al. [16] utilized a fixed point method to derive deformed mean of an $n$-variable mean by a 2 -variable operator mean. They discussed various AndoHiai type inequalities for deformed means. For more information on the deformed means, the readers are referred to $[14,15,31]$.

In this paper, as a continuation of [17], for an $n$-tuple of positive invertible operators on a Hilbert space, we prove some variants of Ando-Hiai type inequalities for the deformed mean from an $n$-variable operator mean by an operator mean, which is related to the information monotonicity of a certain unital positive linear map.

As an application, we discuss the monotonicity of the power mean from the deformed mean in terms of the generalized Kantorovich constants under the operator order. Moreover, we improve the norm inequality for the operator power means related to the Log-Euclidean mean in terms of the Specht ratio. 


\section{DEFORMED MEAN}

Throughout the paper, $\mathbb{B}(\mathcal{H})$ is the $C^{*}$-algebra of all bounded linear operators on a Hilbert space $\mathcal{H} ; \mathbb{B}(\mathcal{H})^{+}$is the cone of positive operators in $\mathbb{B}(\mathcal{H})$; and $\mathbb{P}=\mathbb{P}(\mathcal{H})$ is the set of positive invertible operators in $\mathbb{B}(\mathcal{H})$. For self-adjoint operators $X$ and $Y$, we write $Y \geq X$ (the operator order) if $Y-X$ is positive, and $Y>X$ if $Y-X$ is positive invertible. We denote by $\|X\|_{\infty}$ the operator norm of $X \in \mathbb{B}(\mathcal{H})$. Moreover, we denote by $I$ the identity operator on $\mathcal{H}$ and by SOT the strong operator topology on $\mathbb{B}(\mathcal{H})$.

The notion of 2-variable operator means was introduced by Kubo and Ando [18] in an axiomatic way as follows: A map $\sigma: \mathbb{B}(\mathcal{H})^{+} \times \mathbb{B}(\mathcal{H})^{+} \mapsto \mathbb{B}(\mathcal{H})^{+}$is called an operator mean if it satisfies the following properties:

(i) Monotonicity: $A \leq C, B \leq D \Longrightarrow A \sigma B \leq C \sigma D$.

(ii) Transformer inequality: $C(A \sigma B) C \leq(C A C) \sigma(C B C)$ for every $C \in$ $\mathbb{B}(\mathcal{H})^{+}$.

(iii) Downward continuity: $A_{k} \searrow A, B_{k} \searrow B \Longrightarrow A_{k} \sigma B_{k} \searrow A \sigma B$, where $A_{k} \searrow A$ means that $A_{1} \geq A_{2} \geq \cdots$ and $A_{k} \rightarrow A$ in SOT.

(iv) Normalization: $I \sigma I=I$.

The most important result of [18] gives a one-to-one correspondence $\sigma \leftrightarrow f$ between operator means $\sigma$ and nonnegative operator monotone functions $f$ on $(0, \infty)$ with $f(1)=1$ via

$$
\begin{aligned}
& f(x) I=I \sigma(x I) \quad \text { for } x>0, \\
& A \sigma B=A^{1 / 2} f\left(A^{-1 / 2} B A^{-1 / 2}\right) A^{1 / 2} \quad \text { for } A, B \in \mathbb{P} .
\end{aligned}
$$

The corresponding operator monotone function $f$ to $\sigma$ is said to be the representing function of $\sigma$ and is denoted by $f_{\sigma}$.

To extend operator means to several variables, Hiai et al.[16] consider a map $\mathfrak{M}: \mathbb{P}^{n} \mapsto \mathbb{P}$ as an $n$-variable operator mean if it satisfies:

(I) Monotonicity: If $A_{j}, B_{j} \in \mathbb{P}$ and $A_{j} \leq B_{j}$ for $1 \leq j \leq n$, then

$$
\mathfrak{M}\left(A_{1}, \ldots, A_{n}\right) \leq \mathfrak{M}\left(B_{1}, \ldots, B_{n}\right) .
$$

(II) Congruence invariance: For every $A_{1}, \ldots, A_{n} \in \mathbb{P}$ and any invertible $S \in$ $\mathbb{B}(\mathcal{H})$,

$$
S^{*} \mathfrak{M}\left(A_{1}, \ldots, A_{n}\right) S=\mathfrak{M}\left(S^{*} A_{1} S, \ldots, S^{*} A_{n} S\right)
$$


In particular, the homogeneity holds: $\mathfrak{M}\left(t A_{1}, \ldots, t A_{n}\right)=t \mathfrak{M}\left(A_{1}, \ldots, A_{n}\right)$ for $t>0$.

(III) Monotone continuity: Let $A_{j}, A_{j k} \in \mathbb{P}$ for $1 \leq j \leq n$ and $k \in \mathbb{N}$. If either $A_{j k} \nearrow A_{j}$ or $A_{j k} \searrow A_{j}$ as $k \rightarrow \infty$ for each $j$, then

$$
\mathfrak{M}\left(A_{1 k}, \ldots, A_{n k}\right) \rightarrow \mathfrak{M}\left(A_{1}, \ldots, A_{n}\right) \quad \text { in SOT. }
$$

(IV) Normalized condition: $\mathfrak{M}(I, \ldots, I)=I$.

Assume that $\sigma$ is a 2-variable operator mean such that $\sigma$ is not the left trivial mean $\ell$ defined by $A \ell B=A$. If $\mathfrak{M}$ is an $n$-variable operator mean, then the deformed mean $\mathfrak{M}_{\sigma}$ from $\mathfrak{M}$ by $\sigma$ is defined in [16, Theorem 2.1] to be the unique positive solution of the operator equation

$$
X=\mathfrak{M}\left(X \sigma A_{1}, \ldots, X \sigma A_{n}\right) \quad \text { for } X \in \mathbb{P},
$$

for all positive invertible operators $A_{1}, \ldots, A_{n} \in \mathbb{P}$, or equivalently

$$
I=\mathfrak{M}\left(f_{\sigma}\left(X^{-1 / 2} A_{1} X^{-1 / 2}\right), \ldots, f_{\sigma}\left(X^{-1 / 2} A_{n} X^{-1 / 2}\right)\right),
$$

where $f_{\sigma}$ is the representing function of $\sigma$. Then $\mathfrak{M}_{\sigma}: \mathbb{P}^{n} \mapsto \mathbb{P}$ is an $n$-variable operator mean satisfying (I)-(IV) again. Moreover, if $Y \in \mathbb{P}$ and $Y \leq \mathfrak{M}\left(Y \sigma A_{1}, \ldots, Y \sigma A_{n}\right)$, then $Y \leq \mathfrak{M}_{\sigma}\left(A_{1}, \ldots, A_{n}\right)$. If $Y \in \mathbb{P}$ and $Y \geq \mathfrak{M}\left(Y \sigma A_{1}, \ldots, Y \sigma A_{n}\right)$, then $Y \geq$ $\mathfrak{M}_{\sigma}\left(A_{1}, \ldots, A_{n}\right)$.

For every 2-variable operator mean $\sigma$, it associates another operator mean $\sigma^{*}$ defined by $A \sigma^{*} B=\left(A^{-1} \sigma B^{-1}\right)^{-1}$. $\sigma^{*}$ is called the adjoint mean of $\sigma$. Similarly, for an $n$-variable mean $\mathfrak{M}$, the adjoint mean $\mathfrak{M}^{*}$ is defined by

$$
\mathfrak{M}^{*}\left(A_{1}, \ldots, A_{n}\right)=\mathfrak{M}\left(A_{1}^{-1}, \ldots, A_{n}^{-1}\right)^{-1}, \quad A_{j} \in \mathbb{P} .
$$

Clearly $\mathfrak{M}^{*}$ is itself a mean satisfying (I)-(IV) and $\left(\mathfrak{M}_{\sigma}\right)^{*}=\left(\mathfrak{M}^{*}\right)_{\sigma^{*}}$ for any operator mean $\sigma \neq \ell$.

The following result shows that the deformed mean satisfies the information monotonicity, which is already shown under a more general setting by Hiai and Lim [15], and under a unital case by Pálfia [22].

Theorem 2.1. Let $\Phi: \mathbb{B}(\mathcal{H}) \mapsto \mathbb{B}(\mathcal{K})$ be a normal positive linear map such that $\Phi(I)$ is invertible and let $\sigma$ be a 2-variable operator mean with $\sigma \neq \ell$, which is used in the related constructions to be sure that the corresponding monotone function $f_{\sigma}$ 
is strictly monotone on $(0, \infty)$. If $\mathfrak{M}: \mathbb{P}^{n} \mapsto \mathbb{P}$ is an $n$-variable operator mean with the information monotonicity

$$
\Phi\left(\mathfrak{M}\left(A_{1}, \ldots, A_{n}\right)\right) \leq \mathfrak{M}\left(\Phi\left(A_{1}\right), \ldots, \Phi\left(A_{n}\right)\right)
$$

then the deformed mean $\mathfrak{M}_{\sigma}$ satisfies the information monotonicity:

$$
\Phi\left(\mathfrak{M}_{\sigma}\left(A_{1}, \ldots, A_{n}\right)\right) \leq \mathfrak{M}_{\sigma}\left(\Phi\left(A_{1}\right), \ldots, \Phi\left(A_{n}\right)\right)
$$

Let $\omega=\left(\omega_{1}, \ldots, \omega_{n}\right)$ be a probability vector, that is, $\omega_{j} \geq 0$ and $\sum_{j=1}^{n} \omega_{j}=1$. The weighted arithmetic mean $\mathcal{A}_{\omega}$ and the weighted harmonic mean $\mathcal{H}_{\omega}=\left(\mathcal{A}_{\omega}\right)^{*}$ are defined by

$$
\mathcal{A}_{\omega}=\sum_{j=1}^{n} \omega_{j} A_{j} \quad \text { and } \quad \mathcal{H}_{\omega}=\left(\sum_{j=1}^{n} \omega_{j} A_{j}^{-1}\right)^{-1}
$$

for $A_{1}, \ldots, A_{n} \in \mathbb{P}$. Clearly $\mathcal{A}_{\omega}$ and $\mathcal{H}_{\omega}$ satisfy (I)-(IV).

Let $\mathfrak{M}: \mathbb{P}^{n} \mapsto \mathbb{P}$ be an $n$-variable operator mean with

$$
\mathcal{H}_{\omega} \leq \mathfrak{M} \leq \mathcal{A}_{\omega}
$$

for some probability vector $\omega=\left(\omega_{1}, \ldots, \omega_{n}\right)$. Then for any operator mean $\sigma$, it follows that $\mathfrak{M}_{\sigma}$ satisfies (2.2) again: In fact, put $X=\mathfrak{M}_{\sigma}\left(A_{1}, \ldots, A_{n}\right)$. It follows from $[13,(3.3 .2)]$ that $\alpha_{0}:=f_{\sigma}^{\prime}(1) \in(0,1]$ and so $!_{\alpha_{0}} \leq \sigma \leq \nabla_{\alpha_{0}}$. Hence, the monotonicity of $\mathfrak{M}$ gives

$$
\mathfrak{M}\left(X !_{\alpha_{0}} A_{1}, \ldots, X !_{\alpha_{0}} A_{n}\right) \leq X \leq \mathfrak{M}\left(X \nabla_{\alpha_{0}} A_{1}, \ldots, X \nabla_{\alpha_{0}} A_{n}\right)
$$

and the assumption $\mathcal{H}_{\omega} \leq \mathfrak{M} \leq \mathcal{A}_{\omega}$ implies

$$
\mathcal{H}_{\omega} \leq \mathfrak{M}_{\sigma} \leq \mathcal{A}_{\omega}
$$

If $\mathfrak{M}$ satisfies (2.2), then so does $\mathfrak{M}^{*}$, and hence $\left(\mathfrak{M}^{*}\right)_{\sigma}$ satisfies $(2.2)$, too.

Replacing $A_{j}$ by $A_{j}^{p}$ for $p>0$ in (2.3) and taking the logarithm of both sides, we get

$\log \left(\sum_{j=1}^{n} \omega_{j} A_{j}^{-p}\right)^{-1 / p} \leq \log \mathfrak{M}_{\sigma}\left(A_{1}^{p}, \ldots, A_{n}^{p}\right)^{1 / p} \leq \log \left(\sum_{j=1}^{n} \omega_{j} A_{j}^{p}\right)^{1 / p} \quad$ for all $p>0$,

which gives the Lie-Trotter formula for the deformed mean $\mathfrak{M}_{\sigma}$ as

$$
\lim _{p \rightarrow 0} \mathfrak{M}_{\sigma}\left(A_{1}^{p}, \ldots, A_{n}^{p}\right)^{1 / p}=\exp \left(\sum_{j=1}^{n} \omega_{j} \log A_{j}\right)
$$


Let $\Phi: \mathbb{B}(\mathcal{H}) \mapsto \mathbb{B}(\mathcal{K})$ be a unital positive linear map and let $\omega=\left(\omega_{1}, \ldots, \omega_{n}\right)$ be a probability vector. Then a unital positive linear map $\Psi_{\Phi, \omega}: \mathbb{B}(\mathcal{H}) \oplus \cdots \oplus \mathbb{B}(\mathcal{H}) \mapsto$ $\mathbb{B}(\mathcal{K})$ is defined by

$$
\Psi_{\Phi, \omega}(X)=\sum_{j=1}^{n} \omega_{j} \Phi\left(A_{j}\right)
$$

for $X=A_{1} \oplus \cdots \oplus A_{n}$ and $A_{1}, \ldots, A_{n} \in \mathbb{P}$. If $\Phi$ is the identity map, then we denote it by $\Psi_{\omega}=\Psi_{i d, \omega}$.

In the next theorem, by the Mond-Pečarić method [11, Chapter 1], we show a reverse to the information monotonicity (2.1) without the information monotonicity condition for $\mathfrak{M}$ :

Theorem 2.2. Let $A_{1}, \ldots, A_{n} \in \mathbb{P}$ such that $m I \leq A_{j} \leq M I \quad(j=1, \ldots, n)$ for some scalars $0<m<M$ and let $\Phi: \mathbb{B}(\mathcal{H}) \mapsto \mathbb{B}(\mathcal{K})$ be a unital positive linear map. Assume that $\mathfrak{M}: \mathbb{P}^{n} \mapsto \mathbb{P}$ is an n-variable operator mean satisfying $\mathcal{H}_{\omega} \leq \mathfrak{M} \leq \mathcal{A}_{\omega}$ for some probability vector $\omega=\left(\omega_{1}, \ldots, \omega_{n}\right)$. If $\sigma$ is an operator mean, then

$$
\mathfrak{M}_{\sigma}\left(\Phi\left(A_{1}\right), \ldots, \Phi\left(A_{n}\right)\right) \leq \alpha \Phi\left(\mathfrak{M}_{\sigma}\left(A_{1}, \ldots, A_{n}\right)\right)+\beta(m, M, \alpha) I,
$$

for each $\alpha>0$, where

$$
\beta(m, M, \alpha)= \begin{cases}M+m-2 \sqrt{\alpha M m} & \text { if } m \leq \sqrt{\alpha M m} \leq M, \\ (1-\alpha) m & \text { if } M \leq \sqrt{\alpha M m}, \\ (1-\alpha) M & \text { if } \sqrt{\alpha M m} \leq m .\end{cases}
$$

Proof. By the convexity of $f(t)=t^{-1}$, we have $A^{-1} \leq-\frac{1}{M m} A+\frac{M+m}{M m} I$ and hence

$$
\Phi\left(A^{-1}\right) \leq-\frac{1}{M m} \Phi(A)+\frac{M+m}{M m} I .
$$

Therefore it follows that

$$
\Phi(A)-\alpha \Phi\left(A^{-1}\right)^{-1} \leq \Phi(A)-\alpha\left(-\frac{1}{M m} \Phi(A)+\frac{M+m}{M m} I\right)^{-1} .
$$

Assume that the function $F(t)=t-\alpha\left(\frac{M+m-t}{M m}\right)^{-1}$ is defined on $[m, M]$. Then it follows that $F^{\prime}(t)=0$ has exactly one solution $t_{0}=M+m-\sqrt{\alpha M m}$. From $F^{\prime \prime}\left(t_{0}\right)=2 \sqrt{m M \alpha}>0$, we observe that if $m \leq t_{0} \leq M$, then $\beta(m, M, \alpha)=$ $\max _{m \leq t \leq M} F(t)=F\left(t_{0}\right)=M+m-2 \sqrt{\alpha M m}$. Moreover, $F^{\prime \prime}(t)>0$ implies that if $t_{0} \geq M$, then $\beta(m, M, \alpha)=F(M)=(1-\alpha) M$ and if $t_{0} \leq m$, then $\beta(m, M, \alpha)=$ $f(m)=(1-\alpha) m$. Hence,

$$
\Phi(A)-\alpha \Phi\left(A^{-1}\right)^{-1} \leq \beta(m, M, \alpha) I \quad \text { for each } \alpha>0 .
$$


Since $\mathcal{H}_{\omega} \leq \mathfrak{M} \leq \mathcal{A}_{\omega}$ and $\sigma$ is an operator mean, we have $\mathcal{H}_{\omega} \leq \mathfrak{M}_{\sigma} \leq \mathcal{A}_{\omega}$ and so

$$
\begin{aligned}
\mathfrak{M}_{\sigma}\left(\Phi\left(A_{1}\right), \ldots, \Phi\left(A_{n}\right)\right) & -\alpha \Phi\left(\mathfrak{M}_{\sigma}\left(A_{1}, \ldots, A_{n}\right)\right) \\
& \leq \sum_{j=1}^{n} \omega_{j} \Phi\left(A_{j}\right)-\alpha \Phi\left(\left(\sum_{j=1}^{n} \omega_{j} A_{j}^{-1}\right)^{-1}\right) \\
& \leq \sum_{j=1}^{n} \omega_{j} \Phi\left(A_{j}\right)-\alpha\left(\sum_{j=1}^{n} \omega_{j} \Phi\left(A_{j}^{-1}\right)\right)^{-1} \\
& =\Psi_{\Phi, \omega}(X)-\alpha \Psi_{\Phi, \omega}\left(X^{-1}\right)^{-1} \\
& \leq \beta(m, M, \alpha) I \quad \text { by }(2.7),
\end{aligned}
$$

where $X=A_{1} \oplus \cdots \oplus A_{n}$ and $\Psi_{\Phi, \omega}$ is defined by (2.5).

In particular, we have the following ratio type and difference type reverse inequalities of (2.1).

Corollary 2.3. Under the assumptions as in Theorem 2.2, it follows that

$$
\mathfrak{M}_{\sigma}\left(\Phi\left(A_{1}\right), \ldots, \Phi\left(A_{n}\right)\right) \leq \frac{(M+m)^{2}}{4 M m} \Phi\left(\mathfrak{M}_{\sigma}\left(A_{1}, \ldots, A_{n}\right)\right)
$$

and

$$
\mathfrak{M}_{\sigma}\left(\Phi\left(A_{1}\right), \ldots, \Phi\left(A_{n}\right)\right) \leq \Phi\left(\mathfrak{M}_{\sigma}\left(A_{1}, \ldots, A_{n}\right)\right)+(\sqrt{M}-\sqrt{m})^{2} I .
$$

Proof. If we choose $\alpha$ such that $\beta(m, M, \alpha)=0$ in (2.6), then $\alpha$ coincides with the Kantorovich constant $\frac{(M+m)^{2}}{4 M m}$ and we have (2.8). If we put $\alpha=1$ in (2.6), then $\beta(m, M, 1)=(\sqrt{M}-\sqrt{m})^{2}$ and we have (2.9).

\section{Ando-Hiai type inequalities}

Assume that $\mathfrak{M}: \mathbb{P}^{n} \mapsto \mathbb{P}$ is an $n$-variable operator mean with conditions (I)-(IV) in previous section. For $A_{1}, \ldots, A_{n} \in \mathbb{P}$ we consider Ando-Hiai type inequalities for $\mathfrak{M}$ as follows:

$$
\mathfrak{M}\left(A_{1}^{r}, \ldots, A_{n}^{r}\right) \leq\left\|\mathfrak{M}\left(A_{1}, \ldots, A_{n}\right)\right\|_{\infty}^{r-1} \mathfrak{M}\left(A_{1}, \ldots, A_{n}\right) \quad \text { for all } r \geq 1,
$$

and

$$
\mathfrak{M}\left(A_{1}^{r}, \ldots, A_{n}^{r}\right) \geq\left\|\mathfrak{M}\left(A_{1}, \ldots, A_{n}\right)\right\|_{\infty}^{r-1} \mathfrak{M}\left(A_{1}, \ldots, A_{n}\right) \quad \text { for all } 0<r \leq 1 .
$$

For example, it follows from [16, Lemma 3.2 and Corollary 3.4] that the weighted harmonic mean $\mathcal{H}_{\omega}$ and the Karcher mean $G_{\omega}$ satisfy (3.1). 
A two variable operator mean $\sigma$ is called power monotone increasing (p.m.i) if $f_{\sigma}\left(x^{r}\right) \geq f_{\sigma}(x)^{r}$ for all $x>0$ and $r \geq 1$, see [27].

In [16, Theorem 3.1], Hiai et al. showed several Ando-Hiai type inequalities for $n$-variable operator means of operators: Let $\sigma$ be a p.m.i operator mean with $\sigma \neq \ell$ and let $\mathfrak{M}: \mathbb{P}^{n} \mapsto \mathbb{P}$ be an $n$-variable operator mean. If $\mathfrak{M}$ satisfies (3.1) (resp. (3.2)) for every $A_{1}, \ldots, A_{n} \in \mathbb{P}$, then $\mathfrak{M}_{\sigma}$ satisfies too.

Though we have no relation between $\mathfrak{M}_{\sigma}\left(A_{1}^{r}, \ldots, A_{n}^{r}\right)$ and $\mathfrak{M}_{\sigma}\left(A_{1}, \ldots, A_{n}\right)^{r}$ under the operator order for all $r>0$ in general, Ando-Hiai type inequalities induce the following norm inequality: If $\mathfrak{M}$ satisfies (3.1) (resp. (3.2)), then for any p.m.i operator means with $\sigma \neq \ell$, we have

$$
\left\|\mathfrak{M}_{\sigma}\left(A_{1}^{r}, \ldots, A_{n}^{r}\right)\right\|_{\infty} \leq\left\|\mathfrak{M}_{\sigma}\left(A_{1}, \ldots, A_{n}\right)\right\|_{\infty}^{r} \quad \text { for all } r \geq 1
$$

(resp.

$$
\left.\left\|\mathfrak{M}_{\sigma}\left(A_{1}^{r}, \ldots, A_{n}^{r}\right)\right\|_{\infty} \geq\left\|\mathfrak{M}_{\sigma}\left(A_{1}, \ldots, A_{n}\right)\right\|_{\infty}^{r} \quad \text { for all } 0<r \leq 1 .\right)
$$

In this section, for every unital positive linear map $\Phi$ and every operator mean $\sigma$, we estimate the operator order relations between two deformed means $\mathfrak{M}_{\sigma}\left(\Phi\left(A_{1}^{r}\right), \ldots, \Phi\left(A_{n}^{r}\right)\right)$ and $\Phi\left(\mathfrak{M}_{\sigma}\left(A_{1}, \ldots, A_{n}\right)^{r}\right)$ for $r>0$ in terms of the generalized Kantorovich constant.

To give our main results, we need some preliminaries. We recall that a unital positive linear mapping $\Phi$ satisfies the Davis-Choi-Jensen type inequality

$$
\Phi\left(X^{r}\right) \leq \Phi(X)^{r}
$$

for every $r \in(0,1)$ and $X \in \mathbb{P}$. If $r \in(-1,0) \cup(1,2)$, then a reverse inequality holds in (3.3). Counterpart to this, we recall the next lemma, which shows some upper and lower bounds for the difference and ratio of Jensen type inequalities for power functions.

Lemma 3.1. [11, Theorem 3.16] Let $\Phi: \mathbb{B}(\mathcal{H}) \mapsto \mathbb{B}(\mathcal{K})$ be a unital positive linear map and let $X \in \mathbb{P}$ be a positive invertible operator such that $m I \leq X \leq M I$ for some scalars $0<m<M$. If $r \in \mathbb{R} \backslash[0,1]$ (resp. $r \in(0,1)$ ), then

$$
\Phi\left(X^{r}\right)-\alpha \Phi(X)^{r} \leq \gamma(m, M, r, \alpha) I \quad\left(\text { resp. } \quad \Phi\left(X^{r}\right)-\alpha \Phi(X)^{r} \geq \gamma(m, M, r, \alpha) I\right)
$$


for each $\alpha>0$, where

$$
\gamma(m, M, r, \alpha)= \begin{cases}\alpha(r-1)\left(\frac{M^{r}-m^{r}}{\alpha r(M-m)}\right)^{r /(r-1)}+\frac{M m^{r}-m M^{r}}{M-m} \\ (1-\alpha) M^{r} & \text { if } m \leq\left(\frac{M^{r}-m^{r}}{\alpha r(M-m)}\right)^{1 /(r-1)} \leq M, \\ (1-\alpha) m^{r} & \text { if } M \leq\left(\frac{M^{r}-m^{r}}{\alpha r(M-m)}\right)^{1 /(r-1)},\end{cases}
$$

Two special cases of Lemma 3.1 read as the next lemma. If $\alpha=1$, then we write $\gamma(m, M, r)=\gamma(m, M, r, 1)$. Moreover if we choose $\alpha$ such that $\gamma(m, M, r, \alpha)=0$ in (3.4), then $\alpha=K(h, r)$, where

$$
K(h, p)=\frac{h^{p}-h}{(p-1)(h-1)}\left(\frac{p-1}{p} \frac{h^{p}-1}{h^{p}-h}\right)^{p} \quad \text { for } p \in \mathbb{R} \text { and } h>0,
$$

is called the generalized Kantorovich constant and $h=\frac{M}{m}$, see [11, Definition 2.2]. In particular, $K(h, 2)=K(h,-1)=\frac{(h+1)^{2}}{4 h}$ is called the Kantorovich constant.

Lemma 3.2. With the assumptions as in Lemma 3.1, it follows that

$$
\Phi\left(X^{r}\right)-\Phi(X)^{r} \leq \gamma(m, M, r) I \quad \text { and } \quad \Phi\left(X^{r}\right) \leq K(h, r) \Phi(X)^{r}
$$

hold for all $r \in \mathbb{R} \backslash[0,1]$. If $r \in(0,1)$, then reverse inequalities hold.

In the next theorem, we give an Ando-Hiai type inequality for deformed means as well as a difference counterpart to the information monotonicity.

Theorem 3.3. Assume that $\mathfrak{M}: \mathbb{P}^{n} \mapsto \mathbb{P}$ is an n-variable operator mean satisfies $\mathcal{H}_{\omega} \leq \mathfrak{M} \leq \mathcal{A}_{\omega}$ for some probability vector $\omega=\left(\omega_{1}, \ldots, \omega_{n}\right)$ and that $\sigma$ is an operator mean. If $A_{1}, \ldots, A_{n} \in \mathbb{P}$ such that $m I \leq A_{j} \leq M I \quad(j=1, \ldots, n)$ for some scalars $0<m<M$, then for each $\alpha>0$

$$
\mathfrak{M}_{\sigma}\left(\Phi\left(A_{1}^{r}\right), \ldots, \Phi\left(A_{n}^{r}\right)\right) \leq \alpha \Phi\left(\mathfrak{M}_{\sigma}\left(A_{1}, \ldots, A_{n}\right)^{r}\right)+\gamma(1 / M, 1 / m,-r, \alpha) I
$$

for all $r \in(0,1]$ and all unital positive linear maps $\Phi$, where $\gamma(m, M, r, \alpha)$ is defined by (3.4).

Proof. Since $f_{\sigma}^{\prime}(1) \in(0,1]$ and $\mathcal{H}_{\omega} \leq \mathfrak{M} \leq \mathcal{A}_{\omega}$, we have $\mathcal{H}_{\omega} \leq \mathfrak{M}_{\sigma} \leq \mathcal{A}_{\omega}$. Since $t \mapsto t^{r}$ is operator monotone for $r \in(0,1]$, we get $\mathcal{H}_{\omega}^{r} \leq \mathfrak{M}_{\sigma}^{r}$ and so

$$
\Phi\left(\mathfrak{M}_{\sigma}^{r}\right) \geq \Phi\left(\mathcal{H}_{\omega}^{r}\right)=\Phi\left(\left(\sum_{j=1}^{n} \omega_{j} A_{j}^{-1}\right)^{-r}\right) \geq \Phi\left(\sum_{j=1}^{n} \omega_{j} A_{j}^{-1}\right)^{-r}=\left(\sum_{j=1}^{n} \omega_{j} \Phi\left(A_{j}^{-1}\right)\right)^{-r}
$$


where the right inequality follows from the operator convexity of $t \mapsto t^{-r}$ for $-r \in$ $[-1,0)$. Then it follows that

$$
\begin{aligned}
\mathfrak{M}_{\sigma}\left(\Phi\left(A_{1}^{r}\right), \ldots\right. & \left., \Phi\left(A_{n}^{r}\right)\right)-\alpha \Phi\left(\mathfrak{M}_{\sigma}\left(A_{1}, \ldots, A_{n}\right)^{r}\right) \\
& \leq \sum_{j=1}^{n} \omega_{j} \Phi\left(A_{j}^{r}\right)-\alpha \Phi\left(\mathfrak{M}_{\sigma}\left(A_{1}, \ldots, A_{n}\right)^{r}\right) \quad\left(\text { by } \mathfrak{M}_{\sigma} \leq \mathcal{A}_{\omega}\right) \\
& \left.\leq \sum_{j=1}^{n} \omega_{j} \Phi\left(\left(A_{j}^{-1}\right)^{-r}\right)-\alpha\left(\sum_{j=1}^{n} \omega_{j} \Phi\left(A_{j}^{-1}\right)\right)^{-r} \quad \text { (by }(3.7)\right) \\
& =\Psi_{\Phi, \omega}\left(\left(X^{-1}\right)^{-r}\right)-\alpha \Psi_{\Phi, \omega}\left(X^{-1}\right)^{-r} \\
& \leq \gamma(1 / M, 1 / m,-r, \alpha) I \quad \text { (by Lemma 3.1 and }-r \in[-1,0)),
\end{aligned}
$$

where $X=A_{1} \oplus \cdots \oplus A_{n}$ and $\frac{1}{M} \leq X^{-1} \leq \frac{1}{m}$, and $\Psi_{\Phi, \omega}$ is defined by (2.5).

If we put $\alpha=1$ and choose $\alpha$ such that $\gamma(m, M,-r, \alpha)=0$ in Theorem 3.3, then we have the following difference type and ratio type inequalities of (3.6):

Corollary 3.4. Under the same assumption of Theorem 3.3, if $r \in(0,1)$, then

$$
\mathfrak{M}_{\sigma}\left(\Phi\left(A_{1}^{r}\right), \ldots, \Phi\left(A_{n}^{r}\right)\right)-\Phi\left(\mathfrak{M}_{\sigma}\left(A_{1}, \ldots, A_{n}\right)^{r}\right) \leq \gamma(1 / M, 1 / m,-r) I
$$

and

$$
\begin{aligned}
& \frac{4 M m}{(M+m)^{2}} K(h,-r)^{-1} \Phi\left(\mathfrak{M}_{\sigma}\left(A_{1}, \ldots, A_{n}\right)^{r}\right) \leq \mathfrak{M}_{\sigma}\left(\Phi\left(A_{1}^{r}\right), \ldots, \Phi\left(A_{n}^{r}\right)\right) \\
& \leq K(h,-r) \Phi\left(\mathfrak{M}_{\sigma}\left(A_{1}, \ldots, A_{n}\right)^{r}\right),
\end{aligned}
$$

where the generalized Kantorovich constant $K(h, r)$ is defined by (3.5) and $h=M / m$.

Proof. If we put $\alpha=1$ in (3.6), then we have (3.8). If we choose $\alpha$ such that $\gamma(1 / M, 1 / m,-r, \alpha)=0$ in (3.6), then $\alpha=K(h,-r)$ since $\frac{1 / m}{1 / M}=M / m=h$ and we obtain the second inequality of (3.9).

To get the first inequality of (3.9), note that for every positive invertible operator $A$ with $m I \leq A \leq M I$, we have the reverse Choi's inequality [11, Theorem 1.32]

$$
\Phi\left(A^{-1}\right) \leq \frac{(M+m)^{2}}{4 M m} \Phi(A)^{-1} .
$$

Furthermore, since $-r \in(-1,0)$, Lemma 3.2 for a unital positive linear mapping $\Psi_{\omega}$ defined by (2.5) yields that

$$
\sum_{j=1}^{n} \omega_{j} A_{j}^{-r} \leq K(h,-r)\left(\sum_{j=1}^{n} \omega_{j} A_{j}\right)^{-r} .
$$


It follows that

$$
\begin{aligned}
\mathfrak{M}_{\sigma}\left(\Phi\left(A_{1}^{r}\right), \ldots, \Phi\left(A_{n}^{r}\right)\right) & \geq\left(\sum_{j=1}^{n} \omega_{j} \Phi\left(A_{j}^{r}\right)^{-1}\right)^{-1} \quad\left(\text { by } \mathfrak{M}_{\sigma} \geq \mathcal{H}_{\omega}\right) \\
& \geq\left(\sum_{j=1}^{n} \omega_{j} \Phi\left(A_{j}^{-r}\right)\right)^{-1}=\left(\Phi\left(\sum_{j=1}^{n} \omega_{j} A_{j}^{-r}\right)\right)^{-1} \quad \text { (by } \\
& \left.\geq K(h,-r)^{-1} \Phi\left(\left(\sum_{j=1}^{n} \omega_{j} A_{j}\right)^{-r}\right)^{-1} \quad \text { (by }(3.11) \quad \text { (by }(3.10)\right) \\
& \geq K(h,-r)^{-1} \frac{4 M m}{(M+m)^{2}} \Phi\left(\left(\sum_{j=1}^{n} \omega_{j} A_{j}\right)^{r}\right) \\
& \geq \frac{4 M m}{(M+m)^{2}} K(h,-r)^{-1} \Phi\left(\mathfrak{M}_{\sigma}\left(A_{1}, \ldots, A_{n}\right)^{r}\right)
\end{aligned}
$$

and the last inequality comes from $\mathcal{A}_{\omega} \geq \mathfrak{M}_{\sigma}$ and so $\mathcal{A}_{\omega}^{r} \geq \mathfrak{M}_{\sigma}^{r}$ for $r \in(0,1)$.

The Löwner-Heinz theorem states that if $A \geq B \geq 0$, then $A^{p} \geq B^{p}$ for all $p \in$ $[0,1]$. However, $A \geq B$ does not imply $A^{p} \geq B^{p}$ for $p>1$ in general. Related to the Kantorovich inequality, Furuta [10] showed the following order preserving operator inequality: Let $A$ and $B$ be positive operators with $A \geq B \geq 0$ and $M_{1} I \geq A \geq m_{1} I$ or $M_{2} I \geq B \geq m_{2} I$ for some scalars $0<m_{1} \leq M_{1}$ and $0<m_{2} \leq M_{2}$, and put $h_{1}=M_{1} / m_{1}$ and $h_{2}=M_{2} / m_{2}$. Then

$$
B^{p} \leq K\left(h_{1}, p\right) A^{p} \quad \text { for all } p \geq 1
$$

and

$$
B^{p} \leq K\left(h_{2}, p\right) A^{p} \quad \text { for all } p \geq 1 \text {. }
$$

In the next theorem, we present the Ando-Hiai type inequality for deformed means, when $r \geq 1$.

Theorem 3.5. Let $A_{1}, \ldots, A_{n} \in \mathbb{P}$ such that $m I \leq A_{j} \leq M I \quad(j=1, \ldots, n)$ for some scalars $0<m<M$ and let $\Phi$ be a unital positive linear map. Assume that $\mathfrak{M}: \mathbb{P}^{n} \mapsto \mathbb{P}$ is an n-variable operator mean such that $\mathcal{H}_{\omega} \leq \mathfrak{M} \leq \mathcal{A}_{\omega}$ for some probability vector $\omega=\left(\omega_{1}, \ldots, \omega_{n}\right)$, and $\sigma$ is an operator mean. Then for each $\alpha>0$

$$
\mathfrak{M}_{\sigma}\left(\Phi\left(A_{1}^{r}\right), \ldots, \Phi\left(A_{k}^{r}\right)\right) \leq \alpha \Phi\left(\mathfrak{M}_{\sigma}\left(A_{1}, \ldots, A_{k}\right)^{r}\right)+\gamma\left(\frac{1}{M}, \frac{1}{m},-r, \alpha K(h, r)^{-1}\right) I
$$

for all $r \geq 1$, where $\gamma(m, M, r, \alpha)$ is defined by (3.4). 
Proof. Since $f_{\sigma}^{\prime}(1) \in(0,1]$ and $\mathcal{H}_{\omega} \leq \mathfrak{M} \leq \mathcal{A}_{\omega}$, we have $\mathcal{H}_{\omega} \leq \mathfrak{M}_{\sigma} \leq \mathcal{A}_{\omega}$ and $m I \leq \mathfrak{M}_{\sigma} \leq M I$, and so it follows from (3.12) that

$$
\mathcal{H}_{\omega}^{r} \leq K(h, r) \mathfrak{M}_{\sigma}\left(A_{1}, \ldots, A_{k}\right)^{r}
$$

for all $r \geq 1$. Therefore, for each $\alpha>0$

$$
\sum_{i=1}^{k} \omega_{i} A_{i}^{r}-\alpha \mathfrak{M}_{\sigma}\left(A_{1}, \ldots, A_{k}\right)^{r} \leq \sum_{i=1}^{k} \omega_{i} A_{i}^{r}-\alpha K(h, r)^{-1} \mathcal{H}_{\omega}^{r} .
$$

Moreover, Lemma 3.1 with $X=A_{1}^{-1} \oplus \cdots \oplus A_{k}^{-1}$ gives

$$
\begin{aligned}
\sum_{i=1}^{k} \omega_{i} A_{i}^{r}-\alpha K(h, r)^{-1} \mathcal{H}_{\omega}^{r} & =\sum_{i=1}^{k} \omega_{i}\left(A_{i}^{-1}\right)^{-r}-\alpha K(h, r)^{-1}\left(\sum_{i=1}^{k} \omega_{i} A_{i}^{-1}\right)^{-r} \\
& =\Psi_{\omega}\left(X^{-r}\right)-\alpha K(h, r)^{-1} \Psi_{\omega}(X)^{-r} \\
& \leq \gamma\left(\frac{1}{M}, \frac{1}{m},-r, \alpha K(h, r)^{-1}\right) I,
\end{aligned}
$$

where $\Psi_{\omega}$ is defined by (2.5). Combining these two inequalities yields

$$
\sum_{i=1}^{k} \omega_{i} A_{i}^{r}-\alpha \mathfrak{M}_{\sigma}\left(A_{1}, \ldots, A_{k}\right)^{r} \leq \gamma\left(\frac{1}{M}, \frac{1}{m},-r, \alpha K(h, r)^{-1}\right) I .
$$

Hence, for each $\alpha>0$

$$
\begin{aligned}
\mathfrak{M}_{\sigma}\left(\Phi\left(A_{1}^{r}\right), \ldots, \Phi\left(A_{k}^{r}\right)\right)-\alpha \Phi\left(\mathfrak{M}_{\sigma}\left(A_{1}, \ldots, A_{k}\right)^{r}\right) & \leq \sum_{j=1}^{n} \omega_{j} \Phi\left(A_{j}^{r}\right)-\alpha \Phi\left(\mathfrak{M}_{\sigma}\left(A_{1}, \ldots, A_{k}\right)^{r}\right) \\
& =\Phi\left(\sum_{j=1}^{n} \omega_{j} A_{j}^{r}-\alpha \mathfrak{M}_{\sigma}\left(A_{1}, \ldots, A_{k}\right)^{r}\right) \\
& \leq \gamma\left(\frac{1}{M}, \frac{1}{m},-r, \alpha K(h, r)^{-1}\right) I .
\end{aligned}
$$

Corollary 3.6. Under the same assumption of Theorem 3.5, if $r \geq 1$, then

$$
\mathfrak{M}_{\sigma}\left(\Phi\left(A_{1}^{r}\right), \ldots, \Phi\left(A_{k}^{r}\right)\right)-\Phi\left(\mathfrak{M}_{\sigma}\left(A_{1}, \ldots, A_{k}\right)^{r}\right) \leq \gamma\left(\frac{1}{M}, \frac{1}{m},-r, K(h, r)^{-1}\right) I
$$

and

$$
\begin{aligned}
& \left(\frac{\left(M^{r}+m^{r}\right)^{2}}{4 M^{r} m^{r}}\right)^{-1} K(h,-r)^{-1} K(h, r)^{-1} \Phi\left(\mathfrak{M}_{\sigma}\left(A_{1}, \ldots, A_{k}\right)^{r}\right) \\
& \quad \leq \mathfrak{M}_{\sigma}\left(\Phi\left(A_{1}^{r}\right), \ldots, \Phi\left(A_{k}^{r}\right)\right) \leq K(h,-r) K(h, r) \Phi\left(\mathfrak{M}_{\sigma}\left(A_{1}, \ldots, A_{k}\right)^{r}\right) .
\end{aligned}
$$


Proof. If we put $\alpha=1$ in (3.14) of Theorem 3.5, then we have (3.15). Moreover, from the discussion before Lemma 3.2, we know that if $\gamma\left(\frac{1}{M}, \frac{1}{m},-r, \alpha K(h, r)^{-1}\right)=0$, then $\alpha K(h, r)^{-1}=K(h,-r)$ and so $\alpha=K(h, r) K(h,-r)$. Hence, we obtain the second inequality of (3.16) by putting $\gamma\left(\frac{1}{M}, \frac{1}{m},-r, \alpha K(h, r)^{-1}\right)=0$ in (3.14) of Theorem 3.5.

Next, we prove the first inequality of (3.16). It follows from Lemma 3.2 with $X=A_{1} \oplus \cdots \oplus A_{n}$ and $m I \leq X \leq M I$ that

$$
\sum_{j=1}^{n} \omega_{j} A_{j}^{-r}=\Psi_{\omega}\left(X^{-r}\right) \leq K(h,-r) \Psi_{\omega}(X)^{-r}=K(h,-r)\left(\sum_{j=1}^{n} \omega_{j} A_{j}\right)^{-r}
$$

for $-r \leq-1$, and so

$$
\left(\sum_{j=1}^{n} \omega_{j} A_{j}^{-r}\right)^{-1} \geq K(h,-r)^{-1}\left(\sum_{j=1}^{n} \omega_{j} A_{j}\right)^{r}
$$

Applying the reverse Choi's inequality (3.10) with $X=A_{1}^{-r} \oplus \cdots \oplus A_{n}^{-r}$ and noting that $M^{-r} I \leq X \leq m^{-r} I$, we get

$$
\Psi_{\omega}\left(X^{-1}\right) \leq \frac{\left(M^{-r}+m^{-r}\right)^{2}}{4 M^{-r} m^{-r}} \Psi_{\omega}(X)^{-1}=\frac{\left(M^{r}+m^{r}\right)^{2}}{4 M^{r} m^{r}} \Psi_{\omega}(X)^{-1},
$$

where $\Psi_{\omega}$ is defined by $(2.5)$ and so

$$
\left(\Phi\left(\sum_{j=1}^{n} \omega_{j} A_{j}^{-r}\right)\right)^{-1} \geq\left(\frac{\left(M^{r}+m^{r}\right)^{2}}{4 M^{r} m^{r}}\right)^{-1} \Phi\left(\left(\sum_{j=1}^{n} \omega_{j} A_{j}^{-r}\right)^{-1}\right) .
$$

On the other hand, by Choi's inequality, we can write

$$
\left(\Phi\left(\sum_{j=1}^{n} \omega_{j} A_{j}^{-r}\right)\right)^{-1}=\left(\sum_{j=1}^{n} \omega_{j} \Phi\left(A_{j}^{-r}\right)\right)^{-1} \leq\left(\sum_{j=1}^{n} \omega_{j} \Phi\left(A_{j}^{r}\right)^{-1}\right)^{-1} .
$$


Hence, we have

$$
\begin{aligned}
\mathfrak{M}_{\sigma}\left(\Phi\left(A_{1}^{r}\right), \ldots, \Phi\left(A_{n}^{r}\right)\right) & \geq\left(\sum_{j=1}^{n} \omega_{j} \Phi\left(A_{j}^{r}\right)^{-1}\right)^{-1} \\
& =\left(\Phi\left(\sum_{j=1}^{n} \omega_{j} A_{j}^{-r}\right)\right)^{-1} \quad(\text { by }(3.18)) \\
& \geq\left(\frac{\left(M^{r}+m^{r}\right)^{2}}{4 M^{r} m^{r}}\right)^{-1} \Phi\left(\left(\sum_{j=1}^{n} \omega_{j} A_{j}^{-r}\right)^{-1}\right) \quad(\text { by }(3.17)) \\
& \geq\left(\frac{\left(M^{r}+m^{r}\right)^{2}}{4 M^{r} m^{r}}\right)^{-1} K(h,-r)^{-1} \Phi\left(\left(\sum_{j=1}^{n} \omega_{j} A_{j}\right)^{r}\right) \quad(\text { by Lemm } \\
& \geq\left(\frac{\left(M^{r}+m^{r}\right)^{2}}{4 M^{r} m^{r}}\right)^{-1} K(h,-r)^{-1} K(h, r)^{-1} \Phi\left(\mathfrak{M}_{\sigma}\left(A_{1}, \ldots, A_{n}\right)^{r}\right),
\end{aligned}
$$

whence we derive the first inequality of (3.16). Note that the last inequality comes from $\mathfrak{M}_{\sigma} \leq \mathcal{A}_{\omega}$ and so $\mathfrak{M}_{\sigma}^{r} \leq K(h, r) \mathcal{A}_{\omega}^{r}$ for all $r \geq 1$ by (3.12).

It is known that $\mathfrak{M}_{\sigma}\left(A_{1}^{r}, \ldots, A_{n}^{r}\right)$ and $\mathfrak{M}_{\sigma}\left(A_{1}, \ldots, A_{n}\right)^{r}$ have no relation under the operator order for $r \in \mathbb{R}$. As an application of our results, we have the following corollary.

Corollary 3.7. Let $A_{1}, \ldots, A_{n} \in \mathbb{P}$ such that $m I \leq A_{j} \leq M I \quad(j=1, \ldots, n)$ for some scalars $0<m<M$. If $\mathfrak{M}: \mathbb{P}^{n} \mapsto \mathbb{P}$ is an n-variable operator mean such that $\mathcal{H}_{\omega} \leq \mathfrak{M}_{\sigma} \leq \mathcal{A}_{\omega}$ for some probability vector $\omega=\left(\omega_{1}, \ldots, \omega_{n}\right)$, then

$$
\begin{aligned}
K(h,-r)^{-1} \mathfrak{M}_{\sigma}\left(A_{1}, \ldots, A_{n}\right)^{r} & \leq \mathfrak{M}_{\sigma}\left(A_{1}^{r}, \ldots, A_{n}^{r}\right) \\
& \leq K(h,-r) \mathfrak{M}_{\sigma}\left(A_{1}, \ldots, A_{n}\right)^{r}, \quad(r \in(0,1))
\end{aligned}
$$

and

$$
\begin{aligned}
K(h,-r)^{-1} K(h, r)^{-1} \mathfrak{M}_{\sigma}\left(A_{1}, \ldots, A_{k}\right)^{r} & \leq \mathfrak{M}_{\sigma}\left(A_{1}^{r}, \ldots, A_{k}^{r}\right) \\
& \leq K(h,-r) K(h, r) \mathfrak{M}_{\sigma}\left(A_{1}, \ldots, A_{k}\right)^{r} \quad(r \geq 1)
\end{aligned}
$$

for every operator mean $\sigma$. In particular

$$
\mathfrak{M}_{\sigma}\left(A_{1}, \ldots, A_{n}\right) \leq I \quad \text { implies } \quad \mathfrak{M}_{\sigma}\left(A_{1}^{r}, \ldots, A_{n}^{r}\right) \leq K(h,-r) I \quad(r \in(0,1))
$$

and

$$
\mathfrak{M}_{\sigma}\left(A_{1}, \ldots, A_{k}\right) \leq I \quad \text { implies } \quad \mathfrak{M}_{\sigma}\left(A_{1}^{r}, \ldots, A_{k}^{r}\right) \leq K(h,-r) K(h, r) I \quad(r \geq 1) .
$$


Proof. If we put the identity map $\Phi$ in (3.9), then we have the right inequality in (3.19). The right Inequality in (3.20) follows similarly from Corollary 3.6. For the first inequality in (3.19), note that $\mathcal{H}_{\omega} \leq \mathfrak{M}_{\sigma} \leq \mathcal{A}_{\omega}$ and $\mathfrak{M}_{\sigma}^{r} \leq \mathcal{A}_{\omega}^{r}$ for all $r \in(0,1)$. Then

$$
\begin{aligned}
\mathfrak{M}_{\sigma}\left(A_{1}^{r}, \ldots, A_{n}^{r}\right) & \geq\left(\sum_{j=1}^{n} \omega_{j} A_{j}^{-r}\right)^{-1} \\
& \geq K(h,-r)^{-1}\left(\sum_{j=1}^{n} \omega_{j} A_{j}\right)^{r} \\
& \geq K(h,-r)^{-1} \mathfrak{M}_{\sigma}\left(A_{1}, \ldots, A_{n}\right)^{r}
\end{aligned}
$$

To prove the first inequality in (3.20), we have

$$
\begin{aligned}
\mathfrak{M}_{\sigma}\left(A_{1}^{r}, \ldots, A_{n}^{r}\right) & \geq\left(\sum_{j=1}^{n} \omega_{j} A_{j}^{-r}\right)^{-1} \\
& \left.\geq K(h,-r)^{-1}\left(\sum_{j=1}^{n} \omega_{j} A_{j}\right)^{r} \quad \text { (by }(3.11)\right) \\
& \geq K(h,-r)^{-1} K(h, r)^{-1} \mathfrak{M}_{\sigma}\left(A_{1}, \ldots, A_{n}\right)^{r}
\end{aligned}
$$

for $r \geq 1$.

For each $\alpha \in[-1,1] \backslash\{0\}$ the operator power mean $P_{\omega, \alpha}\left(A_{1}, \ldots, A_{n}\right)$ is defined as the unique solution to the equation

$$
\begin{aligned}
& X=\mathcal{A}_{\omega}\left(X \sharp_{\alpha} A_{1}, \ldots, X \sharp_{\alpha} A_{n}\right) \quad \text { for } 0<\alpha<1 \\
& X=\mathcal{H}_{\omega}\left(X \sharp_{-\alpha} A_{1}, \ldots, X \sharp_{-\alpha} A_{n}\right) \quad \text { for }-1<\alpha<0,
\end{aligned}
$$

that is, for $0<\alpha<1$,

$$
P_{\omega, \alpha}=\left(\mathcal{A}_{\omega}\right)_{\sharp \alpha} \quad \text { and } \quad P_{\omega,-\alpha}=\left(\mathcal{H}_{\omega}\right)_{\sharp \alpha}=\left(P_{\omega, \alpha}\right)^{*} .
$$

We can show a reverse Ando-Hiai inequality for the operator power mean $P_{\omega, \alpha}$ in the traditional way:

$$
P_{\omega, \alpha}\left(A_{1}^{r}, \ldots, A_{n}^{r}\right) \leq\left(\frac{(M+m)^{2}}{4 M m}\right)^{r} P_{\omega, \alpha}\left(A_{1}, \ldots, A_{n}\right)^{r} \quad \text { for } 0<r<1
$$


In fact, for $0<r \leq 1$,

$$
\begin{aligned}
P_{\omega, \alpha}\left(A_{1}^{r}, \ldots, A_{n}^{r}\right) & \leq \sum_{j=1}^{n} \omega_{j} A_{j}^{r} \leq\left(\sum_{j=1}^{n} \omega_{j} A_{j}\right)^{r} \\
& \leq\left(\frac{(M+m)^{2}}{4 M m}\left(\sum_{j=1}^{n} \omega_{j} A_{j}^{-1}\right)^{-1}\right)^{r} \\
& \leq\left(\frac{(M+m)^{2}}{4 M m}\right)^{r} P_{\omega, \alpha}\left(A_{1}, \ldots, A_{n}\right)^{r}
\end{aligned}
$$

and we have (3.21).

If we put $\mathfrak{M}=\mathcal{A}_{\omega}$ and $\sigma=\sharp_{\alpha}$ in Corollary 3.7, then $\mathfrak{M}_{\sigma}=\left(\mathcal{A}_{\omega}\right)_{\sharp_{\alpha}}=P_{\omega, \alpha}$ for $0<\alpha<1$ and we have

$$
P_{\omega, \alpha}\left(A_{1}^{r}, \ldots, A_{n}^{r}\right) \leq K(h,-r) P_{\omega, \alpha}\left(A_{1}, \ldots, A_{n}\right)^{r} .
$$

Then inequality (3.22) is an improvement of inequality (3.21). To show it, we need the following lemma.

Lemma 3.8. Let $h \geq 1$. Then the generalized Kantorovich constant has the following property:

$$
K(h,-r) \leq K(h,-1)^{r} \quad \text { for } r \in(0,1)
$$

and

$$
K(h,-r) \geq K(h,-1)^{r} \quad \text { for } r \notin(0,1)
$$

Proof. Put $F(r)=\log K(h,-r)-r \log K(h,-1)$. Since it follows from [26, Theorem 2.3] that $\log K(h,-r)$ is convex for $r \in \mathbb{R}$, and $F(0)=F(1)=0$, we have $F(r)<0$ for $r \in(0,1)$ and $F(r)>0$ for $r \notin(0,1)$.

By Lemma 3.8, we have $K(h,-r)<\left(\frac{(M+m)^{2}}{4 M m}\right)^{r}$ for $0<r<1$, and so inequality (3.22) is an improvement of inequality (3.21).

\section{Norm INEQUALITIES FOR DEFORMED MEANS}

Let $\mathfrak{M}: \mathbb{P}^{n} \mapsto \mathbb{P}$ be an $n$-variable operator mean satisfying (I)-(IV) introduced in Section 2. Assume that $\mathcal{H}_{\omega} \leq \mathfrak{M} \leq \mathcal{A}_{\omega}$ for some probability vector $\omega=\left(\omega_{1}, \ldots, \omega_{n}\right)$ and that $\mathfrak{M}$ satisfies (3.1). Then the Lie-Trotter formula (2.4) for the deformed mean $\mathfrak{M}_{\sigma}$ holds, and $\mathfrak{M}_{\sigma}$ satisfies (3.1) for a p.m.i operator mean $\sigma \neq \ell$. Hence, for every $A_{1}, \ldots, A_{n} \in \mathbb{P},\left\|\mathfrak{M}_{\sigma}\left(A_{1}^{p}, \ldots, A_{n}^{p}\right)\right\|_{\infty}^{1 / p}$ increases to $\left\|\exp \left(\sum_{j=1}^{n} \omega_{j} \log A_{j}\right)\right\|_{\infty}$ as $p \searrow 0$. On the other hand, $\mathfrak{M}_{\sigma}\left(A_{1}^{p}, \ldots, A_{n}^{p}\right)^{1 / p}$ for $p>0$ is not monotone increasing 
under the operator order. In this section, we consider their operator order relations among $\mathfrak{M}_{\sigma}\left(A_{1}^{p}, \ldots, A_{n}^{p}\right)^{1 / p}$ for $p>0$ in terms of the Specht ratio and the generalized Kanotorovich constant.

For this, we recall an important constant due to Specht [24], which estimates the upper bound of the arithmetic mean by the geometric one for positive numbers: For $x_{1}, x_{2}, \ldots, x_{n} \in[m, M]$ with $0<m<M$ and $h=M / m$,

$$
\frac{x_{1}+x_{2}+\cdots+x_{n}}{n} \leq S(h) \sqrt[n]{x_{1} x_{2} \cdots x_{n}}
$$

where the Specht ratio is defined by

$$
S(h)=\frac{(h-1) h^{\frac{1}{h-1}}}{e \log h} \quad(h \neq 1) \quad \text { and } \quad S(1)=1 .
$$

It is known in [11, Theorem 2.56] that $K\left(h^{r}, s / r\right) \mapsto S\left(h^{s}\right)$ as $r \rightarrow 0$.

Firstly, we show the following relation among $\mathfrak{M}_{\sigma}\left(A_{1}^{p}, \ldots, A_{n}^{p}\right)^{1 / p}$ for $p>0$ under the operator order without the p.m.i condition of $\sigma$ :

Theorem 4.1. Let $A_{1}, \ldots, A_{n} \in \mathbb{P}$ such that $m I \leq A_{j} \leq M I \quad(j=1, \ldots, n)$ for some scalars $0<m<M$. Assume that $\mathfrak{M}: \mathbb{P}^{n} \mapsto \mathbb{P}$ is an n-variable operator mean and satisfies $\mathcal{H}_{\omega} \leq \mathfrak{M} \leq \mathcal{A}_{\omega}$ for some probability vector $\omega=\left(\omega_{1}, \ldots, \omega_{n}\right)$ and that $\sigma$ is an operator mean. If $1 \leq q \leq p$, then

$$
\begin{aligned}
K\left(h^{p},-\frac{q}{p}\right)^{-1 / q} \mathfrak{M}_{\sigma}\left(A_{1}^{p}, \ldots, A_{n}^{p}\right)^{1 / p} & \leq \mathfrak{M}_{\sigma}\left(A_{1}^{q}, \ldots, A_{n}^{q}\right)^{1 / q} \\
& \leq K\left(h^{p},-\frac{q}{p}\right)^{1 / q} \mathfrak{M}_{\sigma}\left(A_{1}^{p}, \ldots, A_{n}^{p}\right)^{1 / p} .
\end{aligned}
$$

If $0<q<1$ and $q<p$, then

$$
\begin{aligned}
K\left(h^{q}, 1 / q\right)^{-1} K\left(h^{p},-q / p\right)^{-1 / q} \mathfrak{M}_{\sigma}\left(A_{1}^{p}, \ldots, A_{n}^{p}\right)^{1 / p} \leq \mathfrak{M}_{\sigma}\left(A_{1}^{q}, \ldots, A_{n}^{q}\right)^{1 / q} \\
\quad \leq K\left(h^{q}, 1 / q\right) K\left(h^{p},-q / p\right)^{1 / q} \mathfrak{M}_{\sigma}\left(A_{1}^{p}, \ldots, A_{n}^{p}\right)^{1 / p}
\end{aligned}
$$

where the generalized Kantorovich constant $K(h, p)$ is defined by (3.5) and $h=$ $M / m$. In particular, if $q \rightarrow 0$, then

$$
\begin{gathered}
S(h)^{-1} S\left(h^{p}\right)^{-1 / p} \mathfrak{M}_{\sigma}\left(A_{1}^{p}, \ldots, A_{n}^{p}\right)^{1 / p} \leq \exp \left(\sum_{j=1}^{n} \omega_{j} \log A_{j}\right) \\
\leq S(h) S\left(h^{p}\right)^{1 / p} \mathfrak{M}_{\sigma}\left(A_{1}^{p}, \ldots, A_{n}^{p}\right)^{1 / p}
\end{gathered}
$$

for all $p>0$, where the Specht ratio $S(h)$ is defined by (4.1). 
Proof. We prove the right inequalities. The left ones can be derived similarly. Suppose that $1 \leq q \leq p$. Since $0<q / p \leq 1$, it follows from Corollary 3.7 that

$$
\mathfrak{M}_{\sigma}\left(A_{1}^{q / p}, \ldots, A_{n}^{q / p}\right) \leq K(h,-q / p) \mathfrak{M}_{\sigma}\left(A_{1}, \ldots, A_{n}\right)^{q / p}
$$

Replacing $A_{j}$ by $A_{j}^{p}$ for all $j=1, \ldots, n$, we get

$$
\mathfrak{M}_{\sigma}\left(A_{1}^{q}, \ldots, A_{n}^{q}\right) \leq K\left(h^{p},-q / p\right) \mathfrak{M}_{\sigma}\left(A_{1}^{p}, \ldots, A_{n}^{p}\right)^{q / p}
$$

Since $0<1 / q \leq 1$, by the Löwner-Heinz inequality, we have

$$
\mathfrak{M}_{\sigma}\left(A_{1}^{q}, \ldots, A_{n}^{q}\right)^{1 / q} \leq K\left(h^{p},-q / p\right)^{1 / q} \mathfrak{M}_{\sigma}\left(A_{1}^{p}, \ldots, A_{n}^{p}\right)^{1 / p}
$$

as desired.

Suppose that $0<q<1$ and $q<p$. By the discussion above, we have

$$
\mathfrak{M}_{\sigma}\left(A_{1}^{q}, \ldots, A_{n}^{q}\right) \leq K\left(h^{p},-q / p\right) \mathfrak{M}_{\sigma}\left(A_{1}^{p}, \ldots, A_{n}^{p}\right)^{q / p}
$$

Since $1 / q \geq 1$, it follows from (3.13) that

$$
\mathfrak{M}_{\sigma}\left(A_{1}^{q}, \ldots, A_{n}^{q}\right)^{1 / q} \leq K\left(h^{q}, 1 / q\right) K\left(h^{p},-q / p\right)^{1 / q} \mathfrak{M}_{\sigma}\left(A_{1}^{p}, \ldots, A_{n}^{p}\right)^{1 / p} .
$$

If $q \rightarrow 0$, then $K\left(h^{q}, 1 / q\right) \rightarrow S(h)$ and $K\left(h^{p},-q / p\right)^{1 / q} \rightarrow S\left(h^{p}\right)^{1 / p}$, and thus we have the desired inequality by the Lie-Trotter formula (2.4).

The next theorem gives a monotonicity property for the norm of the deformed means.

Theorem 4.2. Let $A_{1}, \ldots, A_{n} \in \mathbb{P}$ such that $m I \leq A_{j} \leq M I \quad(j=1, \ldots, n)$ for some scalars $0<m<M$. Assume that $\mathfrak{M}: \mathbb{P}^{n} \mapsto \mathbb{P}$ is an $n$-variable operator mean and satisfies $\mathcal{H}_{\omega} \leq \mathfrak{M} \leq \mathcal{A}_{\omega}$ for some probability vector $\omega=\left(\omega_{1}, \ldots, \omega_{n}\right)$ and that $\sigma$ is an operator mean. Then

$$
\begin{aligned}
K\left(h^{p},-q / p\right)^{-1 / q}\left\|\mathfrak{M}_{\sigma}\left(A_{1}^{p}, \ldots, A_{n}^{p}\right)\right\|_{\infty}^{1 / p} & \leq\left\|\mathfrak{M}_{\sigma}\left(A_{1}^{q}, \ldots, A_{n}^{q}\right)\right\|_{\infty}^{1 / q} \\
& \leq K\left(h^{p},-q / p\right)^{1 / q}\left\|\mathfrak{M}_{\sigma}\left(A_{1}^{p}, \ldots, A_{n}^{p}\right)\right\|_{\infty}^{1 / p}
\end{aligned}
$$

for all $0<q<p$, where the generalized Kantorovich constant $K(h, p)$ is defined by (3.5) and $h=M / m$. In particular, if $q \rightarrow 0$, then

$$
\begin{aligned}
S\left(h^{p}\right)^{-1 / p}\left\|\mathfrak{M}_{\sigma}\left(A_{1}^{p}, \ldots, A_{n}^{p}\right)\right\|_{\infty}^{1 / p} & \leq\left\|\exp \left(\sum_{j=1}^{n} \omega_{j} \log A_{j}\right)\right\|_{\infty} \\
& \leq S\left(h^{p}\right)^{1 / p}\left\|\mathfrak{M}_{\sigma}\left(A_{1}^{p}, \ldots, A_{n}^{p}\right)\right\|_{\infty}^{1 / p}
\end{aligned}
$$

for all $p>0$, where the Specht ratio $S(h)$ is defined by (4.1). 
Proof. For $0<q<p$, we have $0<q / p<1$ and so it follows from (4.2) and the opposite inequality that

$$
\begin{aligned}
K\left(h^{p},-q / p\right)^{-1} \mathfrak{M}_{\sigma}\left(A_{1}^{p}, \ldots, A_{n}^{p}\right)^{q / p} & \leq \mathfrak{M}_{\sigma}\left(A_{1}^{q}, \ldots, A_{n}^{q}\right) \\
& \leq K\left(h^{p},-q / p\right) \mathfrak{M}_{\sigma}\left(A_{1}^{p}, \ldots, A_{n}^{p}\right)^{q / p}
\end{aligned}
$$

and so

$$
\begin{aligned}
K\left(h^{p},-q / p\right)^{-1}\left\|\mathfrak{M}_{\sigma}\left(A_{1}^{p}, \ldots, A_{n}^{p}\right)^{q / p}\right\|_{\infty}^{q / p} & \leq\left\|\mathfrak{M}_{\sigma}\left(A_{1}^{q}, \ldots, A_{n}^{q}\right)\right\|_{\infty} \\
& \leq K\left(h^{p},-q / p\right)\left\|\mathfrak{M}_{\sigma}\left(A_{1}^{p}, \ldots, A_{n}^{p}\right)\right\|_{\infty}^{q / p}
\end{aligned}
$$

Hence, we arrive at the desired inequality:

$$
\begin{aligned}
K\left(h^{p},-q / p\right)^{-1 / q}\left\|\mathfrak{M}_{\sigma}\left(A_{1}^{p}, \ldots, A_{n}^{p}\right)^{1 / p}\right\|_{\infty}^{1 / p} & \leq\left\|\mathfrak{M}_{\sigma}\left(A_{1}^{q}, \ldots, A_{n}^{q}\right)\right\|_{\infty}^{1 / q} \\
& \leq K\left(h^{p},-q / p\right)^{1 / q}\left\|\mathfrak{M}_{\sigma}\left(A_{1}^{p}, \ldots, A_{n}^{p}\right)\right\|_{\infty}^{1 / p}
\end{aligned}
$$

Let $\mathfrak{M}: \mathbb{P}^{n} \mapsto \mathbb{P}$ be an $n$-variable operator mean such that $\mathcal{H}_{\omega} \leq \mathfrak{M} \leq \mathcal{A}_{\omega}$ for some probability vector $\omega=\left(\omega_{1}, \ldots, \omega_{n}\right)$ and let $\sigma$ an operator mean. Let $A_{1}, \ldots, A_{n} \in \mathbb{P}$ such that $m I \leq A_{j} \leq M I \quad(j=1, \ldots, n)$ for some scalars $0<m<M$ and $h=M / m$. We remark that, by Theorem 4.1, under the operator order it holds that

$$
S(h)^{-2} \mathfrak{M}_{\sigma}\left(A_{1}, \ldots, A_{n}\right) \leq \exp \left(\sum_{j=1}^{n} \omega_{j} \log A_{j}\right) \leq S(h)^{2} \mathfrak{M}_{\sigma}\left(A_{1}, \ldots, A_{n}\right) .
$$

On the other hand, under the norm inequality, we have

$$
S(h)^{-1}\left\|\mathfrak{M}_{\sigma}\left(A_{1}, \ldots, A_{n}\right)\right\|_{\infty} \leq\left\|\exp \left(\sum_{j=1}^{n} \omega_{j} \log A_{j}\right)\right\|_{\infty} \leq S(h)\left\|\mathfrak{M}_{\sigma}\left(A_{1}, \ldots, A_{n}\right)\right\|_{\infty} .
$$

Comparing (4.3) and (4.4) indicates the difference between the operator order and the operator norm in terms of the Specht ratio.

If we put $\mathfrak{M}=\mathcal{H}_{\omega}$ and $\sigma=\sharp_{\alpha}$ in Theorem 4.2 , then $\mathfrak{M}_{\sigma}=\left(\mathcal{H}_{\omega}\right)_{\sharp_{\alpha}}=P_{\omega,-\alpha}$ for $0<\alpha<1$ and we have

$$
\left\|P_{\omega,-\alpha}\left(A_{1}^{q}, \ldots, A_{n}^{q}\right)\right\|_{\infty}^{1 / q} \leq K\left(h^{p},-q / p\right)^{1 / q}\left\|P_{\omega,-\alpha}\left(A_{1}^{p}, \ldots, A_{n}^{p}\right)\right\|_{\infty}^{1 / p}
$$

for all $0<q<p$. If $\alpha \rightarrow 0$, then

$$
\left\|G_{\omega}\left(A_{1}^{q}, \ldots, A_{n}^{q}\right)\right\|_{\infty}^{1 / q} \leq K\left(h^{p},-q / p\right)^{1 / q}\left\|G_{\omega}\left(A_{1}^{p}, \ldots, A_{n}^{p}\right)\right\|_{\infty}^{1 / p}
$$


for all $0<q<p$. Moreover, if $q \rightarrow 0$, then

$$
\left\|\exp \left(\sum_{j=1}^{n} \omega_{j} \log A_{j}\right)\right\|_{\infty} \leq S\left(h^{p}\right)^{1 / p}\left\|G_{\omega}\left(A_{1}^{p}, \ldots, A_{n}^{p}\right)\right\|_{\infty}^{1 / p}
$$

for all $p>0$. In [8, Corollary 4.2], it was shown that

$$
\left\|G_{\omega}\left(A_{1}^{q}, \ldots, A_{n}^{q}\right)\right\|_{\infty}^{1 / q} \leq\left(\frac{\left(M^{p}+m^{p}\right)^{2}}{4 M^{p} m^{p}}\right)^{1 / p}\left\|G_{\omega}\left(A_{1}^{p}, \ldots, A_{n}^{p}\right)\right\|_{\infty}^{1 / p}
$$

for all $p>0$.

If $0<q<p$, then Lemma 3.8 implies that $K\left(h^{p},-q / p\right)<K\left(h^{p},-1\right)^{q / p}=$ $\left(\frac{\left(M^{p}+m^{p}\right)^{2}}{4 M^{p} m^{p}}\right)^{q / p}$ and so

$$
K\left(h^{p},-q / p\right)^{1 / q}<\left(\frac{\left(M^{p}+m^{p}\right)^{2}}{4 M^{p} m^{p}}\right)^{1 / p} .
$$

This ensures that inequality (4.5) is an improvement of (4.6). In addition, note that when $q \rightarrow 0$, inequality (4.7) implies that $S\left(h^{p}\right)^{1 / p}<\left(\frac{\left(M^{p}+m^{p}\right)^{2}}{4 M^{p} m^{p}}\right)^{1 / p}$ for all $p>0$.

Acknowledgement. The third author is partially supported by the Ministry of Education, Science, Sports and Culture, Grant-in-Aid for Scientific Research (C), JSPS KAKENHI Grant Number JP 19K03542.

\section{REFERENCES}

[1] T. Ando and F. Hiai, Log majorization and complementary Golden-Thompson type inequality, Linear Algebra Appl. 197 (1994), 113-131.

[2] T. Ando, C.-K. Li, and R. Mathias, Geometric means, Linear Algebra Appl. 385 (2004), $305-334$.

[3] R. Bhatia, Positive definite matrices, Princeton series and applied mathematics, Princeton (NJ); Princeton University Press, 2007.

[4] R. Bhatia and J. Holbrook, Riemannian geometry and matrix geometric means, Linear Algebra Appl. 413 (2006), 594-618.

[5] M. Fujii, Furuta's inequality and its mean theoretic approach, J. Operator Theory 23 (1990), no. $1,67-72$.

[6] M. Fujii and E. Kamei, Ando-Hiai inequality and Furuta inequality, Linear Algebra Appl. 416 (2006), 541-545.

[7] M. Fujii, J. Mićić Hot, J. Pečarić, and Y. Seo, Recent Developments of Mond-Pečarić Method in Operator Inequalities, Monographs in Inequalities 4, Element, Zagreb, 2012.

[8] J. I. Fujii, Y. Seo, and T. Yamazaki, Norm inequalities for matrix geometric means of positive definite matrices, Linear Multilinear Algebra 64 (2016), 512-526. 
[9] S. Furuichi, Matrix trace inequalities on the Tsallis entropies, JIPAM. J. Inequal. Pure Appl. Math. 9 (2008), no. 1, Article 1, 7pp.

[10] T. Furuta, Operator inequalities associated with Hölder-McCarthy and Kantorovich inequalities, J. Inequal. Appl. 2 (1998), 137-148.

[11] T. Furuta, J. Mićić Hot, J. Pečarić, and Y. Seo, Mond-Pečarić Method in Operator Inequalities, Monographs in Inequalities 1, Element, Zagreb, 2005.

[12] S. Golden, Lower bounds for Helmholtz function, Phys. Rev (2), 137 (1965), B1127-B1128.

[13] F. Hiai, Matrix Analysis: Matrix Monotone Functions, Matrix Means, and Majorization, Interdisciplinary Information Sciences, Vol.16, No.2 (2010), 139-248.

[14] F. Hiai, Operator means deformed by a fixed point method, arXiv:1711.10170v1.

[15] F. Hiai and Y. Lim, Operator means of probability measures, arXiv:1901.03858v1.

[16] F. Hiai, Y. Seo, and S. Wada, Ando-Hiai type inequalities for multivariate operator means, Linear Multilinear Algebra, Online: http://www.tandfonline.com/loi/glma20.

[17] M. Kian, M. S. Moslehian, and Y. Seo, Variants of Ando-Hiai inequality for operator power means, Linear Multilinear Algebra, Online: http://doi.org/10.1080/03081087.2019.1635981.

[18] F. Kubo and T. Ando, Means of positive linear operators, Math. Ann. 246 (1980), 205-224.

[19] J. Lawson and Y. Lim, Karcher means and Karcher equations of positive definite operators, Trans. Amer. Math. Soc. Series B 1 (2014), 1-22.

[20] Y. Lim and M. Pálfia, Matrix power means and the Karcher mean, J. Funct. Anal. 262 (2012), $1498-1514$.

[21] M. Moakher, A differential geometric approach to the geometric mean of symmetric positivedefinite matrices, SIAM J. Matrix Anal. Appl. 26 (2005), 735-747.

[22] M. Pálfia, Operator means of probability measures and generalized Karcher equations, Adv. Math. 289 (2016), 951-1007.

[23] Y. Seo, Matrix trace inequalities on Tsallis relative entropy of negative order, J. Math. Anal. Appl. 472 (2019), no. 2, 1499-1508.

[24] W. Specht, Zur Theorie der elementaren Mittel, Math. Z. 74, 91-98, (1960)

[25] A. C. Thompson, On certain contraction mappings in a partially ordered vector space, Proc. Amer. Math. Soc. 14 (1963), 438-443.

[26] M. Tominaga, Estimates of Ando-Hiai type inequalities on operator power means, preprint.

[27] S. Wada, Some ways of constructing Furuta-type inequalities, Linear Algebra Appl. 457 (2014), $276-286$.

[28] S. Wada, When does Ando-Hiai inequality hold?, Linear Algebra Appl. 540 (2018), 234-243.

[29] T. Yamazaki, An extension of Kantorovich inequality to $n$-operators via the geometric mean by Ando-Li-Mathias, Linear Algebra Appl. 416 (2006), 688-695.

[30] T. Yamazaki, The Riemannian mean and matrix inequalities related to the Ando-Hiai inequality and chaotic order, Oper. Matrices 6 (2012), 577-588.

[31] T. Yamazaki, The Ando-Hiai inequalities for the solution of the generalized Karcher equation and related results, J. Math. Anal. Appl. 479 (2019), 531-545. 
1 Department of Mathematics, University of Bojnord, P.O. Box 1339, Bojnord 94531, IRAN

E-mail address: kian@ub.ac.ir

2 Department of Pure Mathematics, Center of Excellence in Analysis on Algebraic Structures (CEAAS), Ferdowsi University of Mashhad, P. O. Box 1159, MASHHAD 91775, IRAN.

E-mail address: moslehian@um.ac.ir; moslehian@yahoo.com

3 Department of Mathematics Education, Osaka Kyoiku University, Asahigaoka, KASHIWARA, OSAKA 582-8582, JAPAN.

E-mail address: yukis@cc.osaka-kyoiku.ac.jp 